\title{
EFECTO TÓXICO Y ECOTOXICOLÓGICO DE ARENAS NEGRAS DE LA MINERÍA ARTESANAL EN MADRE DE DIOS
}

\author{
Elsa Huamán Paredes ${ }^{* a}$, Denis Vicuña Puente ${ }^{\mathrm{b}}$, Lizardo Visitación Figueroa ${ }^{\mathrm{a}}$, \\ Lisveth Flores del Pino ${ }^{\mathrm{a}}$
}

\begin{abstract}
RESUMEN
En el presente trabajo se estudió el efecto tóxico y ecotoxicológico de arenas negras (ANM) provenientes de la extracción artesanal de oro, en el departamento de Madre de Dios. Para evaluar el efecto tóxico se usó el test "Toxicity Characteristic Leaching Procedure" (TCLP) y con los lixiviados se hicieron pruebas de ecotoxicidad agudas según el protocolo de la EPA 600/3-88/029 usando microorganismos de prueba, neonatos de Daphnia Magna, con 24 horas de nacidos y en semillas de Lactuca sativa. La cuantificación de mercurio total en las muestras de ANM y en los lixiviados se realizó mediante Espectroscopia de Emisión Atómica de Plasma Acoplado Inductivamente. Los valores obtenidos de mercurio total en las muestras de ANM estuvieron en el rango de 0,1 y $>275 \mathrm{mg} / \mathrm{kg}$ de arena negra, lo que indica que superan en 1000 veces los valores de fondo. En los lixiviados la concentración de mercurio total estuvo en el rango de 0,001 y $>2,75 \mathrm{mg} / \mathrm{L}$, algunos de estos valores superan los límites máximos permisibles $(0,2 \mathrm{mg} / \mathrm{L})$ representado un potencial tóxico para la salud humana. En el caso del efecto ecotóxico sobre las daphnias, se encontró que el metal mercurio en interacción con otros metales, como el aluminio, podrían ocasionar un efecto letal. Sobre la Lactuca sativa, se determinó mayor efecto ecotóxico sobre la radícula.
\end{abstract}

Palabras clave: Arenas negras, mercurio, efectos tóxicos y ecotoxicológicos.

\section{TOXIC AND ECOTOXICOLOGICAL EFFECT OF BLACK SAND OF ARTISANAL MINING IN MADRE DE DIOS}

\begin{abstract}
In this work the toxic and ecotoxicological effect of black sand (ANM) from artisanal gold extraction in the department of Madre de Dios was studied. Toxicity Characteristic Leaching Procedure (TCLP) was used to evaluate the toxic effect and with the leachates,

\footnotetext{
${ }^{a}$ Centro de Investigación en Química, Toxicología y Biotecnología Ambiental, Departamento Académico de Química, Facultad de Ciencias, Universidad Nacional Agraria La Molina, Lima - Perú. ehuaman@lamolina.edu.pe

b Tower and Tower S.A.Av. Portillo Grande Mz. G Lote 10. Lurín, Lima - Perú.
} 
acute ecotoxicity tests were performed according to EPA protocol 600 / 3-88 / 029 using test microorganisms, Daphnia Magna neonates , with 24 hours of birth and in seeds of Lactuca Sativa. The quantification of total mercury in ANM samples and in leachates was performed by Inductively Coupled Plasma Atomic Emission Spectroscopy. The values obtained of total mercury in the ANM samples were in the range of 0.1 and $>275 \mathrm{mg} / \mathrm{kg}$ of black sand indicating that they exceed the background values by 1000 times. In the leachates the total mercury concentration was in the range of 0.001 and $>2.75 \mathrm{mg} / \mathrm{L}$, some of these values exceed the maximum permissible limits $(0.2 \mathrm{mg} / \mathrm{L})$ representing a toxic potential for human health. In the case of the ecotoxic effect on daphnia, it was found that metal mercury in interaction with other metals, such as aluminum, could cause a lethal effect. On the Lactuca Sativa, a greater ecotoxic effect on radicle was determined.

Key words: Black sand, mercury, toxic and ecotoxicological effect

\section{INTRODUCCIÓN}

El proceso de extracción de oro en la región de Madre de Dios - Perú, se realiza en forma artesanal en las cuencas de los ríos Madre de Dios, Inambari, Tambopata, Huaypetue entre otros. Por lo general, para la extracción se utiliza el método gravimétrico, en este método el material de suelo extraído pasa por un tamiz, separando el material grueso constituido por grava, luego el material separado pasa a unos jigs (lavador por sacudidas) y por vibración se logra separar el material pesado denominado arenas negras de Madre de Dios, al que llamaremos ANM (Pastor, 1991). Las ANM son mezcladas con el mercurio para formar la amalgama entre el mercurio y el oro, el mercurio amalgamado con el oro es retirado, quedando las ANM residuales que contienen cantidades variables de mercurio residual y son dispuestas de diversas formas, entre adecuadas e inadecuadas (MINAM, 2011).

Las arenas negras, en general, tienen alta densidad, están conformadas por material pesado que contiene diversos elementos metálicos como el titanio, hierro, aluminio, manganeso, magnesio y calcio (Abdel-Karim et al., 2016), adicionalmente en el caso de ANM el mercurio es el elemento más abundante introducido en forma intencional.

Las concentraciones altas de los elementos prioritarios ( $\mathrm{Sb}, \mathrm{As}, \mathrm{Be}, \mathrm{Cd}, \mathrm{Cr}, \mathrm{Cu}, \mathrm{Pb}, \mathrm{Ag}, \mathrm{Ni}$, Se, Ag, Tl, Zn) según EPA (USEPA 2014) en lixiviados de ANM categorizan a este residuo como residuo peligroso que debe ser manejado y dispuesto según la normativa de residuos sólidos D.L N 1278 , Ley de Gestión Integral de Residuos Sólidos.

La presencia de elementos metálicos potencialmente tóxicos, presentes en las ANM, pueden tener efectos tóxicos cuando son liberados en el ambiente sustancias como el mercurio, pueden generar ecotoxicidad sobre semillas como la de Lactuca sativa, generando formación de fitoquelatinas que restringen el ingreso de nutrientes por la raíz (Cui et al., 2014), por otro lado, la presencia de aluminio en los lixiviados puede ser muy tóxico para los organismos acuáticos como las daphnias, debido a la precipitación en las agallas (Quiroz-Vázquez et al., 2010; Poléo et al., 1994). 
En el Perú son limitados los trabajos realizados sobre arenas negras de la minería de oro (Gómez, 2012; Pastor et al., 1991), motivo por el cual nuestro objetivo para la presente investigación fue evaluar el efecto tóxico y ecotoxicológico de las arenas negras para poder definir su manejo y método de disposición final.

\section{PARTE EXPERIMENTAL}

\section{Toma de muestra}

Las muestras se obtuvieron de forma manual en los lugares de disposición de las arenas negras, luego del proceso de extracción de oro de la minería artesanal en las cercanías del río Madre de Dios y los ríos tributarios del mismo, tal como se muestra en la figura 1.

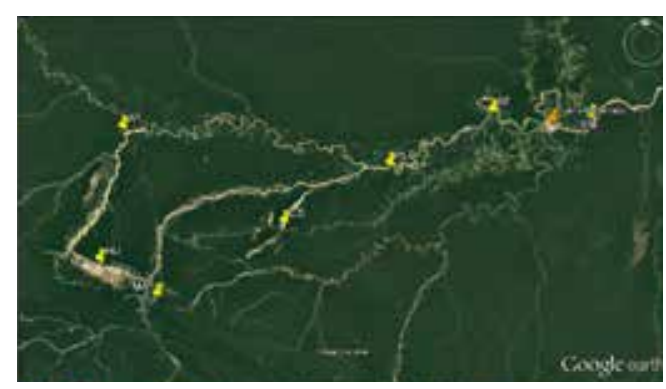

Figura 1. Distribución espacial de los puntos de muestreo. DATUM: UTM WGS84 Zona 19S

Tabla 1. Puntos de muestreo

\begin{tabular}{|c|c|c|c|c|}
\hline \multirow{2}{*}{ Estación } & \multirow{2}{*}{ Cuenca } & \multicolumn{2}{|c|}{ Coordenadas UTM } & \multirow{2}{*}{$\begin{array}{c}\text { Condición } \\
\text { de la } \\
\text { muestra }\end{array}$} \\
\hline & & Este & Norte & \\
\hline A1 & Colorado & 340133 & 8604775 & \\
\hline MA & Inambari & 351756 & 8550941 & $\begin{array}{l}\text { Seca } \\
\text { Fresca }\end{array}$ \\
\hline $3 \mathrm{~F}$ & Madre de Dios & 459808 & 8611242 & Fresca \\
\hline PL & Madre de Dios & 426604 & 8593687 & $\begin{array}{c}\text { Seca } \\
\text { Fresca }\end{array}$ \\
\hline $\mathrm{BM}$ & Bajo Madre de Dios & 491616 & 8609772 & Seca \\
\hline CS & $\begin{array}{l}\text { Tambopata - } \\
\text { Inambari }\end{array}$ & 392682 & 8574898 & Seca \\
\hline $\mathrm{HU}$ & Huaypetue & 333170 & 8562033 & $\begin{array}{c}\text { Seca } \\
\text { Fresca }\end{array}$ \\
\hline
\end{tabular}




\section{Determinación de metales en las arenas negras}

La determinación de metales en los residuos de ANM y en los lixiviados se realizó por Espectrometría de Emisión Atómica de Plasma Acoplado Inductivamente. EPA - Método 200.7 Revisión 4.4.

\section{Determinación del efecto tóxico y ecotoxicológico}

La determinación del efecto tóxico de las ANM se realizó mediante el método EPA 1311 "Toxicity Characteristic Leaching Procedure" (TLCP) utilizando una dilución del residuo 20:1 con un extractante a $\mathrm{pH}=2.2$.

La determinación del efecto ecotoxicológico en los lixiviados de las muestras se realizó utilizando los lineamientos del protocolo de la EPA 600/3-88/029 para test agudos. También se utilizó como organismos de prueba neonatos de Daphnia magna con 24 horas de nacidos, evaluando la concentración que ocasiona un efecto tóxico al $50 \%$ de la población $\left(\mathrm{CE}_{50}\right.$, muerte) durante 24 horas y con semillas de Lactuca sativa evaluando efectos sobre la raíz y la germinación.

Los parámetros ecotoxicológicos fueron transformados en unidades tóxicas (UT), mediante el uso de la fórmula de $U T=\frac{C}{C E_{x}}$, , (US EPA 1992) donde $C$ es la concentración del residuo máxima utilizada (en este caso $100 \%$ ) y $\mathrm{CE}_{\mathrm{x}}$ es la concentración que ocasiona efecto. En el trabajo se utilizó $\mathrm{CE}_{50}$ efecto al $50 \%$ de la población.

\section{Relación entre metales y TCLP en las matrices evaluadas}

Se elaboró la variación del mercurio total en ANM en comparación con la concentración característica de los lixiviados de ANM.

\section{Correlación entre metales y ensayos ecotoxicológicos en las matrices evaluadas}

Se elaboró una matriz de correlación de Pearson entre los elementos metálicos traza presentes en las ANM y los ensayos ecotoxicológicos evaluados en los lixiviados de ANM obtenidos por TCLP.

\section{RESULTADOS Y DISCUSIÓN}

\section{Determinación de metales en las arenas negras}

En la figura 2 se muestra las concentraciones de mercurio presentes en las diferentes estaciones de muestreo y se observa que dos de ellas sobrepasan el nivel de $260 \mathrm{mg} / \mathrm{kg}$, según la Norma de la USEPA, lo que indica claramente el aporte antropogénico de mercurio en las ANM, ratificando lo afirmado por Álvarez et al. (MINAM 2011). 


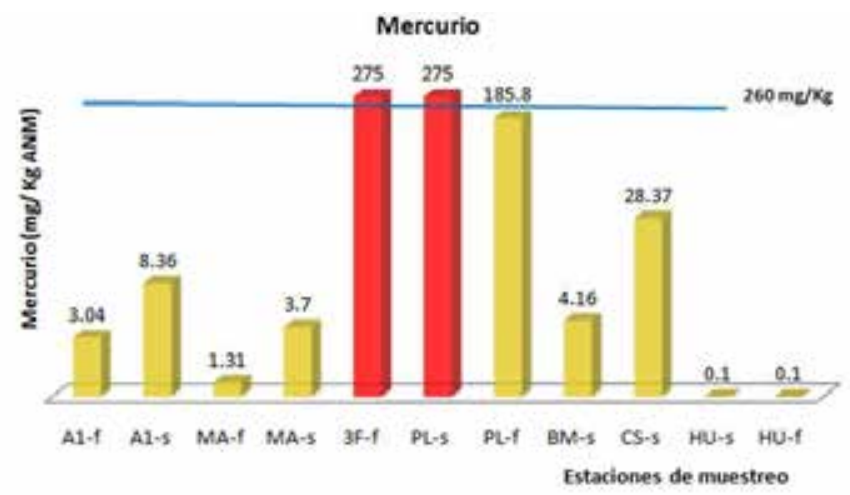

Figura 2. Concentración de mercurio en las muestras de cada estación.

En las muestras analizadas, además del metal mercurio, la presencia del metal aluminio estuvo en mayor proporción frente a otros metales de acuerdo a la figura 3.

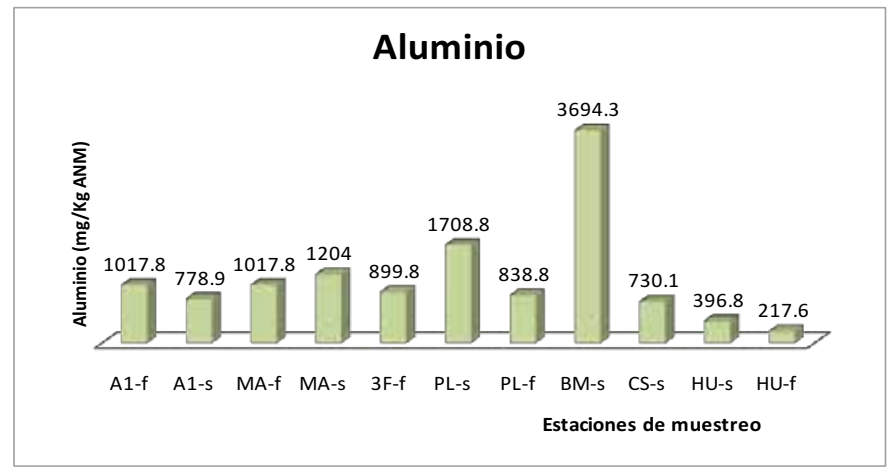

Figura 3. Concentración de aluminio en las muestras

\section{Efecto tóxico en las muestras de ANM}

En los lixiviados, según el método TCLP, el mercurio en la muestra PL-s supera el límite máximo permisible y para el aluminio en la muestra BM-s dio un valor máximo de $10,07 \mathrm{mg} / \mathrm{L}$ que se relaciona con la concentración encontrada en el residuo de ANM En la figura 4 del diagrama de caja y bigotes se evidencia la presencia de estos metales en los residuos de ANM, catalogando a estas muestras como residuos tóxicos que deben ser manejados y dispuestos como residuos peligrosos. 

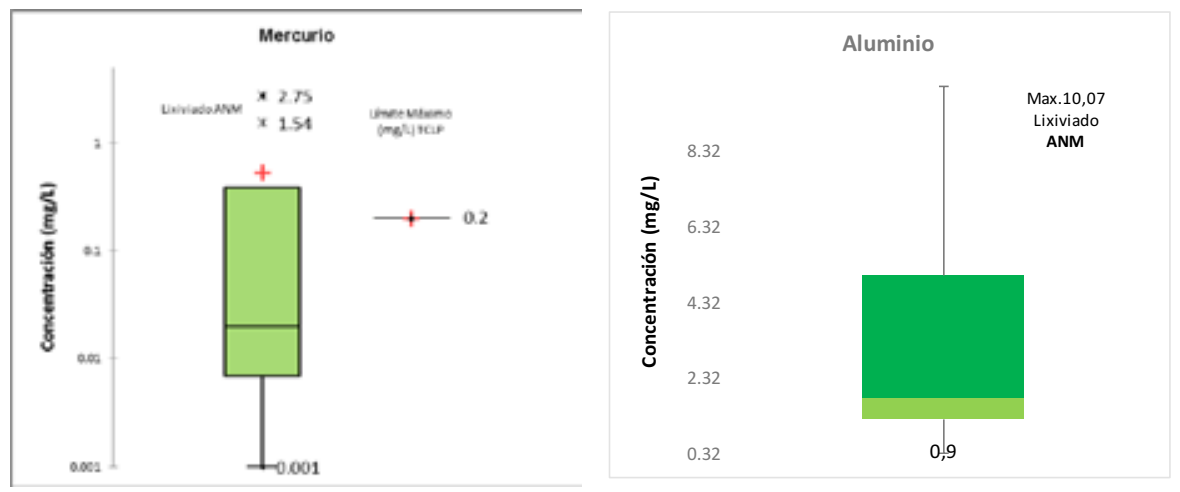

Figura 4. Concentración de mercurio y aluminio en lixiviados de ANM

\section{Correlación entre metal y TCLP en las matrices evaluadas}

Como se observa en la figura 5, en el test de TCLP, muestras con concentraciones de mercurio total en las ANM por debajo de $28,4 \mathrm{mg} / \mathrm{kg}$ presentan contenidos de mercurio en el lixiviado menor a $0,03 \mathrm{mg} / \mathrm{L}$. Cuando la concentración de mercurio se incrementa en las ANM, las concentraciones en el lixiviado se incrementan en forma proporcional a la concentración total. La mayor concentración de mercurio en el lixiviado fue mayor a $2,75 \mathrm{mg} / \mathrm{L}$ en la muestra 3F-F esta muestra también tiene la mayor concentración de mercurio total en la ANM, resultados con tendencias similares han sido encontrados en otros estudios realizados en China (Yang et al., 2016).

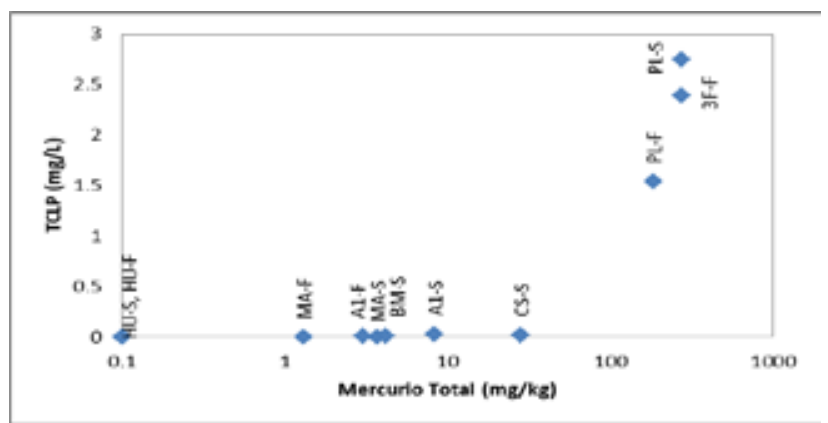

Figura 5. Variación del mercurio total en ANM en comparación con la concentración característica en los lixiviados.

\section{Caracterización ecotoxicológica en Daphnia magna y Lactuca sativa}

En la tabla 2 se presentan los resultados ecotoxicológicos de los lixiviados de ANM. Los valores de ecotoxicidad de los lixiviados fueron convertidos en valores de unidad tóxica (UT) (US EPA, 1992), con la finalidad de poder tener resultados en valores adimensionales que son aditivos y comparables. Los valores de las UT fueron comparados con el promedio 
de toxicidad y utilizando una escala arbitraria (Manusadzianas, 2003; Persoone, 1993), se clasificó la toxicidad de los residuos como: sin toxicidad (st) $<1$ UT, tóxico (t) $1-10$ UT, muy tóxico (mt) 11 - $100 \mathrm{UT}$, extremadamente tóxico (et) > $100 \mathrm{UT}$. Como se observa en la tabla 2, los resultados de las ANM tomaron valores entre muy tóxico a extremadamente tóxicos.

Tabla 2. Unidades tóxicas de los lixiviados de ANM.

\begin{tabular}{|c|c|c|c|c|c|c|c|c|c|}
\hline \multirow{3}{*}{ Muestra } & \multicolumn{4}{|c|}{ EC50 (\%) } & \multicolumn{4}{|c|}{ UT } & \multirow{3}{*}{$\begin{array}{c}\text { UT } \\
\text { Promedio }\end{array}$} \\
\hline & \multicolumn{2}{|c|}{ Daphnia magna } & \multicolumn{2}{|c|}{ Lactuca Sativa } & \multicolumn{2}{|c|}{ Daphnia magna } & \multicolumn{2}{|c|}{ Lactuca sativa } & \\
\hline & $24 \mathrm{~h}$ & $48 \mathrm{~h}$ & Radícula & Germinación & $24 \mathrm{~h}$ & $48 \mathrm{~h}$ & Radícula & Germinación & \\
\hline A1-F & 2,69 & 1,39 & 8,20 & 14,47 & 37,2 & 72,0 & 12,2 & 6,9 & $32,1(\mathrm{mt})$ \\
\hline A1-S & 1,07 & 0,25 & 8,30 & 16,54 & 93,2 & 408,2 & 12,0 & 6,0 & 129,9 (et) \\
\hline MA-F & 0,51 & 0,15 & 8,73 & 16,37 & 196,9 & 653,6 & 11,5 & 6,1 & 217,0 (et) \\
\hline MA-S & 0,56 & 0,18 & 8,10 & 16,60 & 178,6 & 571,4 & 12,3 & 6,0 & $192,1(\mathrm{et})$ \\
\hline $3 \mathrm{~F}-\mathrm{F}$ & 0,73 & 0,21 & 11,99 & 26,41 & 136,6 & 473,9 & 8,3 & 3,8 & $155,7(\mathrm{et})$ \\
\hline PL-F & 1,95 & 1,10 & 6,30 & 18,37 & 51,3 & 90,9 & 15,9 & 5,4 & $40,9(\mathrm{mt})$ \\
\hline PL-S & 1,72 & 0,58 & 5,71 & 14,08 & 58,1 & 173,9 & 17,5 & 7,1 & $64,2(\mathrm{mt})$ \\
\hline BM-S & 1,87 & 0,95 & - - & - - & 53,4 & 105,5 & -- & -- & $79,5(\mathrm{mt})$ \\
\hline $\mathrm{CS}-\mathrm{S}$ & 1,06 & 0,29 & 11,41 & 18,39 & 94,3 & 348,4 & 8,8 & 5,4 & 114,2 (et) \\
\hline
\end{tabular}

En la tabla 3 se correlaciona los resultados ecotoxicológicos en UT con las concentraciones de mercurio y aluminio presentes en las ANM. Se observa que la concentración de aluminio en las ANM tiene una buena correlación con la toxicidad en Daphnia magna tanto a $24 \mathrm{~h}$ como a $48 \mathrm{~h}$. Concentraciones altas de aluminio en soluciones ácidas como los lixiviados de las ANM son muy tóxicas para Daphnia magna, principalmente debido a la precipitación del aluminio en las agallas de los micro crustáceos que actúan como superficies de nucleación donde se polimeriza el aluminio (Quiroz-Vázquez et al., 2010; Poléo et al., 1994), esto puede producir la obstrucción de los espacios interlaminares.

Tabla 3. Coeficientes de correlación de Pearson en Unidades Toxicas (UT)

\begin{tabular}{lcccccc}
\hline \multirow{2}{*}{ Variables } & \multicolumn{2}{c}{ Metales en ANM } & \multicolumn{2}{c}{ Daphnia magna } & \multicolumn{2}{c}{ Lactuca sativa } \\
& $\mathrm{Al}$ & $\mathrm{Hg}$ & $24 \mathrm{~h}$ & $48 \mathrm{~h}$ & Radícula & Germinación \\
\hline $\mathrm{Al}$ & $\mathbf{1}$ & 0,169 & $\mathbf{0 , 6 3 0}$ & $\mathbf{0 , 5 3 6}$ & 0,165 & $-0,056$ \\
$\mathrm{Hg}$ & & $\mathbf{1}$ & $-0,480$ & $-0,532$ & $\mathbf{0 , 8 1 0}$ & 0,270 \\
$\mathrm{D}$ 24 h & & & $\mathbf{1}$ & 0,970 & $-0,450$ & $-0,316$ \\
$\mathrm{D}$ 48 h & & & & $\mathbf{1}$ & $-0,533$ & $-0,340$ \\
LS Radícula & & & & $\mathbf{1}$ & 0,634 \\
LS Germinación & & & & & $\mathbf{1}$ \\
\hline
\end{tabular}

La correlación entre la concentración de mercurio y la ecotoxicidad en Lactuca sativa a nivel de radícula en lixiviados de ANM fue muy buena, esto se debe a que las plantas dificultan el ingreso del mercurio formando complejos de fitoquelatinas con el metal,(Hg-PC) a nivel de la raíz como mecanismo de defensa (Cui et al., 2014), siendo la raíz la que presenta la mejor respuesta ecotoxicológica al mercurio. 


\section{CONCLUSIONES}

Los resultados de análisis de los residuos de arenas negras de Madre de Dios (ANM) presentan contenidos variables de mercurio $(0,1->275 \mathrm{mg} / \mathrm{kg} \mathrm{ANM})$.

Mediante ensayos de toxicidad como el TCLP se ha determinado que las muestras de arenas negras, debido al contenido de mercurio, son residuos peligrosos por superar los valores límite para este metal $(0,2 \mathrm{mg} / \mathrm{L})$.

Las ANM podrían ser consideradas como muy tóxicas a extremadamente tóxicas en los organismos como las daphnias y Lactuca sativa. Principalmente, es el aluminio en copresencia con el mercurio representando toxicidad para las daphnias.

Los residuos de arenas negras son residuos peligrosos que deben ser manejados adecuadamente y dispuestos en rellenos de seguridad, conforme establece la legislación peruana.

Los compuestos alquilmercurio no han sido considerados en este documento sino la cuantificación de mercurio total en las arenas negras de Madre de Dios. Es así que el estudio de especies metiladas o etiladas de mercurio amerita otro análisis.

\section{AGRADECIMIENTO}

El agradecimiento para la realización del proyecto, para la Empresa Tower \& Tower S.A, según el proyecto PITEI-1-P-117-043-14 financiado por FINCyT, aporte propio, y de la Universidad Nacional Agraria La Molina, mediante el uso de laboratorios y equipo.

\section{BIBLIOGRAFÍA}

1. Manusadzianas L, Balkelyte L, Sadauskas K, Blinova I, Põllumaa L, Kahru A. Ecotoxicological study of Lithuanian and Estonian wastewaters: selection of the biotests, and correspondence between toxicity and chemical-based indices. Aquat Toxicol. 2003; 63: 27-41.

2. Persoone G, Goyvaerts M, Janssen C, De Coen W, Vangheluwe M. Cost effective acute hazard monitoring of polluted waters and waste dumps with the aid of Tox kits, Final Report. Commission of European Communities. Contract ACE 89/BE 2/D3. 1993. p. 600.

3. Quiroz-Vázquez P, Sigee DC, White KN. Bioavailability and toxicity of aluminium in a model planktonic food chain (Chlamydomonas-Daphnia) at neutral $\mathrm{pH}$. Limnologica. 2010; 40: 269-277.

4. Poléo ABS, Lydersen E, Rosseland BO, Kroglund F, Salbu B, Vogt RD, et al. Increased mortality of fish due to changing Al-chemistry of mixing zones between limed streams and acidic tributaries. Water Air Soil Pollut. 1994, 75: 339-351. 
5. Gómez CF. Evaluación de escenarios alternativos en sistemas sociales ecológicos afectados por la minería aluvial en Madre de Dios. [Tesis para optar el grado de Magíster of Science]. Lima: Universidad Nacional Agraria La Molina; 2014.

6. Cui L, Feng X, Lin C, Wang X, Meng B, Wang H. Accumulation and translocation of $198 \mathrm{Hg}$ in four crop species. Environ Toxicol Chem. 2014; 33(2): 334-340.

7. Yang M, Wang S, Zhang L, Wu Q, Wang F, Hui M, et al. Mercury emission and speciation from industrial gold production using roasting process. J Geochem Explor. 2016; 117:72-77.

8. Taylor SR. Abundance of chemical elements in the continental crust: a new table. Geochim Cosmochim Acta. 1964; 28(8):1273 -1285.

9. Pastor O. Gisementsalluviauxd'or, 1991, 1-5. Pastor O. El oro en la cuenca del río Madre de Dios - Perú. En: Hérail G, Fornari M. Simposio Internacional sobre Yacimientos Aluviales de Oro; 1-5 jun 1991; La Paz. La Paz: Orstom; 1991. P. 103-113.

10. Brack A, Ipenza C, Alvarez J, Sotero V; Minería Aurífera en Madre de Dios y Contaminación con Mercurio - Una Bomba de Tiempo. Lima: Ministerio del Ambiente; 2011.

11. Abdel-Karim AM, Zaid SM, Moustafa MI, Barakat MG. Mineralogy, chemistry and radioactivity of the heavy minerals in the black sands, along the northern coast of Egypt. J Afr Earth Sci. 2016; 123: 10-20.

12. USEPA. Priority Pollutant List. [Internet]. 2014, United State Environmental Protection Agency. [Revisado jun 2017]. Disponible en: https://www.epa.gov/sites/production/ files/2015-09/documents/priority-pollutant-list-epa.pdf

13. USEPA. Introduction to Water Quality-Based Toxics Control for the NPDES Program. Washington: USEPA; 1992.

14. Coelho JP, Monteiro RJR, Catry T, Lourenco PM, Catry P, Regalla A. et al. Estimation of mercury background values in sediment and biota of the Bijagós archipelago, GuineaBissau. Mar Pollut Bull. 2016; 111: 488-492.

15. Nesbitt HW, Young GM, Bosman SA. Major and trace element geochemistry and genesis of supracrustal rocks of the North Spirit Lake Greenstone belt, NW Ontario, Canada. Precambr Res. 2009; 174: 16-34.

16. Park JW, Hu Z, Gao S, Campbell IH, Gong H. Platinum group element abundances in the upper continental crust revisited - New constraints from analyses of Chinese loess. Geochim Cosmochim Acta. 2012; 93: 63-76.

17. Ji X, Hu W, Cheng J, Yuan T, Fang X, Qu L, et al. Oxidative stress on domestic ducks (Shaoxing duck) chronically exposed in a Mercury-Selenium coexisting mining area in China. Ecotoxicol Environ Saf. 2005; 64: 171-177.

18. Wang W, Hao W, Bian Z, Lei S, Wang X, Sang S, et al. Effect of coal mining activities on the environment of Tetraena mongolica in Wuhai, Inner Mongolia, China-A geochemical perspective. Int J Coal Geol. 2014; 132: 94-102. 